\title{
Template Selection by Editing Algorithms: A Case Study in Face Recognition
}

\author{
Biagio Freni, Gian Luca Marcialis, and Fabio Roli \\ University of Cagliari \\ Department of Electrical and Electronic Engineering \\ Piazza d'Armi - I-09123 Cagliari (Italy) \\ \{biagio.freni, marcialis, roli\}@diee.unica.it
}

\begin{abstract}
In this paper, we consider the problem of template selection in biometric systems as analogous to the problem, called "editing", of selecting representative prototypes when using the 1-Nearest Neighbour classifier (NN). Four editing algorithms are used and compared by experiments with state-of-the-art template selection algorithms. Experiments are performed on a benchmark face data set. Reported results show pros and cons of editing algorithms for template selection in biometric systems.
\end{abstract}

Keywords: Nearest neighbour, biometrics, face recognition, template selection.

\section{Introduction}

Biometrics are aimed to recognize or verify the personal identity by physiological or behavioral characteristics [1. Among others, widely used biometrics are the fingerprint and the face. The core of a biometric system is the so-called gallery, made up of a set of at least one template, which is the feature set extracted, for example, from the face image of a certain person, collected during the enrolment session. Template(s) is stored into the system and it is used, by computing its "distance" from the input sample released by the person during the system operation, in order to authenticate, or recognize, his/her identity. As it can be easily noticed, the system performance (the recognition accuracy, or the tradeoff between false and true authentications) is dependent on the score, that is, on the representativeness degree of the template for each identity (or client, as it is usually named in verification applications).

So far, only Uludag et al. addressed the problem of selecting templates for each client. They proposed some algorithms derived from the clustering theory. they selected, from each gallery, the best samples according to a given criterion, which can be summarised in: maximum similarity among templates, in order to point out their common characteristics, and minimum similarity among them, in order to capture very different characteristics of the same client (intra-class variations). Worth noting, algorithms proposed by Uludag et al. selected the best templates by looking only at the instances collected of the given client, 
without analysing the representativeness of the template with respect to the others. Another issue for these algorithms is that they aren't able to find the number of templates for a certain client. This is a setting parameter given by the human expert, and does not take into account the intrinsic "difficulty" in classifying certain clients with respect to others. Furthermore, no generalization ability is estimated.

As alternative, we believe that the problem of template selection [2] can be dealt by considering its analogies with the "editing" of training sets in Nearest Neighoubour-based classification (NN) [356/7/8,9]. Editing consists in generating, from a given training set $T$, a subset $E$ able to maintain the same classification accuracy of $T$ on itself. In our opinion, the editing algorithms adopted for NN-based classification exhibit an interesting peculiarity: they focus on the whole collected training set $T$. Therefore, they are able to obtain the best galleries set $E$ by analysing the whole structure of the data, i.e., all stored clients samples, by re-sizing the gallery for each client appropriately. This should have a twofold impact: (i) the overall analysis of the structure of $T$ should help in finding templates (instances of $T$ ) exhibiting generalization abilities superior than that of algorithms proposed by Uludag et al [2]; (ii) some "difficult" clients could need galleries bigger than those of other "easy" ones, for which, on the contrary, related galleries could be small. In other words, the gallery size should be adapted as function of the client.

Therefore, in this paper, we consider the problem of the template selection as analogous to the problem of finding representative instances sets when using the NN classifier. It can be easily noticed that NN classifier and current approaches for person recognition are correlated, and share some aspects. For example, they are based on the same criteria of "distance from a template" and it is not difficult to see the set of galleries in biometrics as the training set used in NN-based classification. We experimentally compare such editing approaches with the state-of-the-art template selection algorithms proposed in 2. Experimental results support our claims.

The paper is organized as follows. Section 2 introduces the common terminology we used both in general NN editing approaches and biometrics. Section 3 reviews the main general approaches to edit a given training set for NN-based applications, and summarizes the algorithms in [2]. Section 4 presents some experimental results by adopting a face data set. Section 5 concludes the paper.

\section{Terminology and Notation Adopted}

The following terminology has been used in this paper, which, in our opinion, can be commonly shared in NN-based classification and biometrics recognition. In brackets we pointed out the related application field:

- class $(N N) /$ client(biometrics): a set of objects with common characteristics 34. In this paper, the number of classes is indicated with $C$. In personal recognition applications, each client identity corresponds to one class. 
- genuine users/impostors(biometrics): In personal verification applications, two subclasses are defined: the one of clients whose claimed and true identity correspond, named "genuine users", and the opposite one, named "impostors". In this paper, we only dealt with the face recognition application, thus these terms will not be used.

- instance $(N N) /$ template(biometrics): a certain feature set extracted from a given biometric. Although in the NN literature [5], the "instance" and "template" terms do not mean the same thing, in this paper we always considered the template and instance terms with the same substantial meaning. In particular, we indicated as instances the members of the set called $T$, that is, the initial training set of instances, collected during the enrolment session. At least one instance per class is present in $T$. We used the term "template" after the editing process is completed. In other words, with the terms "templates" we referred to the instances selected from $T$ by the editing algorithm. We referred to the unknown input sample to the system with the term sample. The sample is represented in the same feature space of instances.

- label(NN,biometrics): simply the class identifier. For example, if we have $C$ classes, we can indicate the set of labels as $\Omega=\left\{\omega_{1}, \ldots, \omega_{C}\right\}$. In other words, a labeling function $l(*)$ is given such that, for each $x \in T, l(x)=\omega$, with $\omega \in \Omega$. In biometrics, the label for each class can be the user's name or identification number.

- gallery(biometrics): is the set of possible instances (templates) for a certain client, stored into the memory of the system or embedded into a smart-card. The term "gallery" is typical in biometric applications.

- distance(NN)/score(biometrics): they are quite similar, and exhibit nonnegative output values. The only difference is that a small value of the distance function means that the instance/sample are "close", thus quite similar. Instead, a small value of the similarity function means that they are "far", so quite different. It will be possible to use indifferently distance or similarity function after an appropriate normalization [10. In biometrics, the matching score can be derived from a given distance.

\section{K-NN Editing for Biometrics and State-of-the-Art}

The K-Nearest Neighbour classifier (KNN) belongs to the category of nonparametric classifiers 4 4. Given a sample $x$, this classifier exploit a training set $T$ of templates (instances) in order to find the $\mathrm{K}$ nearest neighbours templates on the basis of a pre-defined distance (or similarity) function.

The weak point of the KNN classifier is the high computational complexity. The more is the size of $T$, the more is the computation amount required. This is an issue in many real classification problems [3]. If a large amount of data are available in $T$, many of them could be redundant or outliers. Therefore, a possible solution to reduce the complexity of $\mathrm{KNN}$ is to find the most representative instances in the training set. This approach is called "editing". Several editing algorithms have been proposed so far $5[6 / 7 / 899$. 
Result of these algorithms is the so-called "edited" set $E \subseteq T$, satisfying some requirements given by the algorithm applied. This edited set must be representative of $T$. The manner to reach this aim can be roughly described as incremental, if $E$ is initially empty and starts growing according to some specific criteria; decremental, if $E$ is initially equal to $T$ and instances are iteratively deleted until a certain stop criterion is met.

In this paper, we only focus the case of $K=1$, thus adopting the simple Nearest Neighbour algorithm.

Key points of the editing algorithms are: the distance or similarity function used, the selection criterion of the "best" instances in $T$, the stop criterion adopted. The distance or similarity function in this kind of application have a dramatic effect. In principle, such function impact on the classification accuracy, thus on the "nearest neighbours" of a given sample, or instance. In this paper we used the simple Euclidean Distance, but the selection of the best distance function is an open problem, although in biometrics it is simplified due to the fact that, often, a dedicated function is designed for the feature selected (e.g. String algorithm for fingerprint matching [12]). The selection and stop criteria depend on the specific algorithm as described in the following.

\subsection{The Proposed Algorithms}

CNN - Condensed NN: Aim of this incremental algorithm is to find an edited set $E \subseteq T$ such that the closest istance $y \in T$ to a given instance $x \in E$ exhibits the same class identifier of $x$. In this way, the maximum classification accuracy on $T$ is reached [6]. Pseudo-code:

1. $C$ instances $x_{1}, \ldots, x_{C}$ are initially selected, such that $l\left(x_{i}\right)=\omega_{i}$ (one instance per class).

2. $E \leftarrow\left\{x_{1}, \ldots, x_{C}\right\}$.

3. $T \leftarrow T-E$.

4. $T$ is classified using $E$ as training set.

5. Let $Y$ the set of misclassified instances in $T$.

6. if $Y \neq \phi$ then
(a) $E \leftarrow E \cup Y$.
(b) $T \leftarrow T-Y$.
(c) Repeat from 4

\section{Stop.}

SNN - Selective NN: This algorithm is an extended version of the previous one [7]. In particular, the aim of this algorithm is to build an Edited set $E$ as small as possible. According to this, not all the misclassified instances were added to $E$, but that who as much close to the own class than any other instance of different class. Therefore, SNN does not guarantee the $100 \%$ of classification accuracy on $T$. Since this algorithm is a clear extension of CNN, we omitted its pseudo-code for sake of space.

RNN - Reduced NN: This is a decremental algorithm. It starts with $E$ set as $T$. Instances are iteratively deleted from $E$ if their deletion does not imply 
a misclassification on $T$. This algorithm stops when no other instance can be removed from $E$ [8]. Pseudo-code:

1. $E \leftarrow T$.

2. for each $x \in E$
(a) $E \leftarrow E-\{x\}$
(b) Classify $T$ using $E$
(c) Let $Y$ be the set of misclassified samples in $T$
(d) if $Y \neq \phi$ then $E \leftarrow E \cup\{x\}$

ENN - Edited NN: This algorithm also starts with an Edited set $E$ equal to $T$ [9]. Instances are removed from $E$, if do not agree with the majority of its "k-nearest neighbour" (in our case $\mathrm{k}=3$ ). Pseudo-code:

1. $E \leftarrow T$.

2. for each $x \in E$

(a) Let $Y$ the set of "k nearest neighbour" instances of $x$.

(b) Let $m$ the majority class label of the instances in $Y$.

(c) Let $l(x)$ the class label of $x$.

(d) if $l(x) \neq m$ then $E \leftarrow E-\{x\}$

\subsection{Editing Approaches vs. State-of-the-Art}

The above algorithms have been experimentally compared with the ones proposed in [2], and named MDIST and DEND. These algorithms are described in the following.

MDIST: The rationale behind the MDIST algorithm comes from the distancebased clustering 11. The aim is to build galleries made up of very close instances, for each client.

A very important parameter for this algorithm is the number of instances, namely, $N$, to select for each class. In 2 this number was an input parameter given by the user.

1. $E \leftarrow \phi$.

2. for each $\omega \in \Omega$
(a) $T^{\omega} \leftarrow\{x \in T: l(x)==\omega\}$
(b) $S \leftarrow\left\{S_{1}, \ldots, S_{k}\right\}$ such that $\left|S_{i}\right|==N$ and $S_{i} \leftarrow\left\{x \in T^{\omega}\right\}$
(c) for $i=0, \ldots, k$
i. $d_{i} \leftarrow$ average distance among instances in $S_{i}$
(d) $S_{\min } \leftarrow\left\{S_{i}: i=\operatorname{argmin}_{j} d_{j}\right\}$
(e) $E \leftarrow E \bigcup S_{\min }$

DEND: The basic idea of this algorithm comes also from the clustering theory (in particular, from the hierarchical clustering [11]). The selection criteria of this algorithm is opposite to the previous one: instead of the selection of very close instances, this algorithm selects the farest instances from a given gallery.

The rationale of DEND is to build galleries able to represent very large intraclass variations of each client. 
1. $E \leftarrow \phi$.

2. for each $\omega \in \Omega$

(a) $T^{\omega} \leftarrow\{x \in T: l(x)==\omega\}$

(b) $S \leftarrow\left\{S_{1}, \ldots, S_{k}\right\}$ such that $\left|S_{i}\right|==N$ and $S_{i} \leftarrow\left\{x \in T^{\omega}\right\}$

(c) for $i=0, \ldots, k$

i. $d_{i} \leftarrow$ average distance among instances in $S_{i}$

(d) $S_{\max } \leftarrow\left\{S_{i}: i=\operatorname{argmax}_{j} d_{j}\right\}$

(e) $E \leftarrow E \bigcup S_{\max }$

A first critical analysis can be done after the description of state-of-the-art and proposed algorithms. Firstly, as stated in the Introduction, MDIST and DEND perform the search of best templates only by analysing the instances of each client. Proposed algorithms, instead, look for best templates by investigating the whole instance space. This can be an advantage, especially with respect to the DEND algorithm. Since it tries to find the largest intra-class variations, the result could be affected by some outliers, or noisy instances which could be very near to other clients. Secondly, the number of templates per gallery $N$ is a fundamental parameter for MDIST and DEND. So far, no methods have been proposed to estimate such number. Therefore, a certain range of $N$ values must be investigated. This problem does not affect the editing algorithms. On the other hand, it can be noticed that the result of incremental approaches (CNN and SNN) is quite dependent on the initial instances selected. Therefore, obtained gallery size can be unstable if outliers are selected. Moreover, decremental approaches starts from the whole $T$, and this could lead to a weak gallery size reduction. Finally, the stop criterion is strongly correlated with the effectiveness of both incremental and decremental algorithms. For example, CNN can reach $100 \%$ of accuracy on $T$ only at the last iteration, before meeting the stop condition. If this one is altered, selected templates could be not representative enough.

\section{Experimental Results}

\subsection{Data Set and Experimental Protocol}

We adopted the Equinox Face Dataset [13. In particular, our experiments are carried out on 50 clients randomly chosen, each one made up of 100 images. Therefore, the data set size is 5000 images.

Each image used was first cropped and, after histogram stretching and normalization, re-sized by keeping the intra-ocular distance on a constant value. This is a common practice in face recognition and verification applications [13. The final size of each image is 100x100 pixels.

In our experiments we adopted the protocol below:

1. The overall data set has been randomly partitioned in two parts of the same size, which we named $T$ and $t$. Thus, each client/class is made up of 50 instances collected during the so-called "enrolment" operation, that is, the registration of each client into the system.

2. PCA [3] was performed on $T$ and $t$ was transformed accordingly, in order to reduce the size of the feature space. 
Table 1. Mean and, in brackets, standard deviation of number of templates per class in $E$ (second column), and of the recognition accuracy of investigated editing approaches (third column). The first column reported the gallery related to the algorithm adopted. The term "TRAIN" means that the overall $T$ is used for classification. Performance of MDIST and DEND has been reported in the case of six and nine instances per client. The best trade-off between recognition accuracy and galleries size has been highlighted in bold.

\begin{tabular}{|c|c|c|}
\hline Gallery & \#instances $\times$ class & TEST \\
\hline TRAIN & $50(0)$ & $99.62(0.14)$ \\
\hline CNN & $\mathbf{7 ( 3 )}$ & $\mathbf{9 7 . 6 ( 0 . 4 5 )}$ \\
\hline SNN & $4(3)$ & $73.66(3.31)$ \\
\hline RNN & $17(9)$ & $98.43(0.53)$ \\
\hline ENN & $49(1)$ & $99.35(0.27)$ \\
\hline MDIST & $6(0)$ & $94.15(0.68)$ \\
\hline MDIST & $9(0)$ & $96.56(0.58)$ \\
\hline DEND & $6(0)$ & $89.11(1.39)$ \\
\hline DEND & $9(0)$ & $94.03(0.70)$ \\
\hline
\end{tabular}

3. $T$ has been used as training set to be edited, by adopting proposed algorithms, MDIST and DEND. All algorithm led to a different edited set $E$, containing the "best" galleries according to the related method.

4. Performance of each approach has been estimated on $t$ set, thus evaluating the generalization ability of the obtained gallery.

5. Items 1-3 were repeated for six runs.

6. Results have been averaged and reported in the following tables and plots.

The performance parameter adopted is the recognition accuracy when the 1-Nearest Neighbour is selected for classification.

\subsection{Results}

First of all, we verified if it is possible, by selecting templates with proposed editing algorithms, to obtain a generalization accuracy better than that achieved with state-of-the-art algorithms based on clustering, as MDIST and DEND.

In table 11. the average size of galleries in $E$, and the mean and standard deviation of the recognition accuracy were shown. Performance of MDIST and DEND has been reported in the case of six and nine instances per client.

From Table 1 the following observations can be drawn:

1. Incremental approaches (SNN and $\mathrm{CNN}$ ) allowed a galleries size reduction much superior than decremental ones, without significant loss in accuracy. The exception is for SNN algorithm which exhibited an accuracy worse than that of other algorithms, due to its stringent working conditions: in fact, the galleries found by SNN have the smallest size.

2. Best accuracy is guarantee by decremental approaches (RNN and ENN) but at the expense of the reduction of the galleries size. 
Table 2. Average recognition accuracy for classes for which the editing has built galleries larger than that of MDIST and DEND. It can be noted that the average accuracy is lower than that in Table 1 for such classes (MIDST and DEND columns), thus they can be classified as "difficult". On the other hand, the editing algorithms allowed to found a gallery large enough, thus allowing a better accuracy.

\begin{tabular}{|c|c|c|c|c|c|c|c|}
\hline Gallery & \#classes & \#instances $\times$ class & TEST & MDIST 9 & DEND 9 & MDIST 6 & DEND 6 \\
\hline CNN & 8 & 12 & 96.18 & 93.78 & 87.56 & 89.41 & 77.26 \\
\hline RNN & 41 & 20 & 98.58 & 96.05 & 93.25 & 93.20 & 87.89 \\
\hline ENN & 50 & 49 & 99.35 & 96.56 & 94.03 & 94.15 & 89.11 \\
\hline
\end{tabular}

Table 3. Average recognition accuracy for classes for which the editing has built galleries smaller than that of MDIST and DEND. It can be noted that the accuracy is about the same as the one reported by MDIST and DEND, thus those classes can be classified as intrinsically "easy".

\begin{tabular}{|c|c|c|c|c|c|c|c|}
\hline Gallery & \#classes & \#instances $\times$ class & TEST & MDIST 9 & DEND 9 & MDIST 6 & DEND 6 \\
\hline CNN & 21 & 4 & 98.04 & 98.46 & 96.88 & 96.89 & 93.67 \\
\hline SNN & 31 & 3 & 60.70 & 96.86 & 95.06 & 94.33 & 90.89 \\
\hline RNN & 4 & 3 & 98.08 & 99.60 & 98.00 & 98.96 & 95.76 \\
\hline
\end{tabular}

3. MDIST and DEND confirmed their strong dependence of the number of istances set, which is not simple to find a priori, and can be given as a constraint, relying, for example, on the maximum recognition time or memory available for storing galleries.

4. On the overall, CNN appeared to be the best trade-off between performance achieved and size of the galleries.

We also investigated if the gallery size of editing algorithms can be considered as function of the instrinsic difficulty of clients. In order to confirm or deny this claim, we proceeded as follows: (i) we isolated all clients for which the editing algorithm found a gallery larger than that of MDIST and DEND with $N=9$; (ii) we also isolated all clients for which a gallery smaller than that of MDIST and DEND with $N=6$ have been found by the editing algorithms. We expected that, for "difficult" clients, related to item (i), the average accuracy is higher for the proposed approaches, whilst it is about the same of state-of-the-art algorithms for "easy" clients of item (ii). Results related to "difficult" clients have been reported in Table 2, whilst Table 3 shows accuracy of "easy" clients.

In the case of Table 2, the SNN performance has not been reported since this algorithm strongly reduce the size of the galleries: only one client following constraints of item (i) was found. The opposite holds for the ENN algorithm in Table 3. No clients with a gallery size less than six were found (item (ii)).

By observing the other algorithms, it can be easily noticed that results confirmed what we expected. For "difficult" clients, the editing algorithms exhibited an accuracy more than that of state-of-the-art algorithm. Both incremental and decremental approaches were effective. The same can be noticed for "easy" 
clients: in this case the editing algorithms pointed out two important advantages: the gallery size is small, and the accuracy is unaltered. This has a positive effect when stringent conditions are required for galleries, especially in terms of the memory available for storing galleries or the matching time.

\subsection{Discussion on Results}

Reported results clearly showed that editing algorithms can be considered as a good alternative to the state-of-the-art approaches to the template selection: they allowed to select templates with good generalization ability and their number is modelled according to the characteristics of each client.

We believe that both features are correlated to the fact that the editing algorithms analysed the overall structure of the data, so they are able to exploit an amount of information larger than that exploited by MDIST and DEND.

On the other hand, some issues must be considered when using these algorithms for the practical selection of templates. A noticeable problem is how to limit the gallery size when strong requirements are present. For example, if the number of templates per clients can not exceed a certain value due to memory size or matching time constraints. By adopting incremental approaches, this limitation can be easily overcame: the increase of the number of templates for a client may stop when the limit is reached. However, it is worth noting that performing such an early stopping could strongly impact on the obtained galleries. A possible solution, which could work when using both incremental or decremental approaches, could be the joint use of them and state-of-the-art ones. Since the template editing approaches allow to find "easy" and "difficult" clients, a first pre-processing of the training set could be done by editing algorithms. Next, the DEND algorithm could be applied to the obtained galleries whose size exceeded the limit. We suggested DEND because it selects the most representative intraclass variations among templates found by editing algorithms. This could also allow to overcome the problem of DEND of selecting outliers or instances too near to the ones of other clients, thanks to the preliminary analysis performed by editing. The above suggestions need further experimental investigations to be confirmed or denied, thus the problem is still open.

Another aspect to point out is that these algorithms can be used when the number of collected instances per class has become large enough, since a large analysis on the data structure is necessary. This may be problematic for certain biometric applications (as the personal verification), where usually a large number of samples can be collected only after long periods of time. A possible solution could be to exploit samples collected during the system operations, and classified with very high matching score. This "semi-supervised" way of improving galleries have been also proposed in the literature [14, but disregarding the editing approaches.

\section{Conclusions}

In this paper, the problem of selecting templates from samples collected during one or multiple enrolment sessions has been treated, by considering its analogies 
with the problem of "editing" a training set in Nearest Neighbour-based classification. We applied the editing algorithms in the context of face recognition.

In particular, four NN-based editing algorithms have been compared with two state-of-the-art algorithms based on the clustering theory. Preliminary results showed that reported algorithms can find a gallery size such that overall accuracy is kept high, whilst the number of comparison per person notably decreased. The reported performance, in some cases, is better than that achieved by state-ofthe-art algorithms, especially by considering that the best number of templates per class is fixed in such approaches.

Although this limited set of experiments is not enough to draw definitive conclusions, we believe that editing approaches for the templates selection could be considered as a good alternative to, or used jointly with, the algorithms so far proposed in the literature.

\section{References}

1. Jain, A.K., Pankanti, S. (eds.): Biometrics: Personal Identification in Networked society. Kluwer Academic Publishers, Dordrecht (1999)

2. Uludag, U., Ross, A., Jain, A.K.: Biometric template selection and update: a case study in fingerprints. Pattern Recognition 37(7), 1533-1542 (2004)

3. Duda, R.O., Hart, P.E., Stork, D.G.: Pattern Classification. John Wiley \& Sons, Chichester (2001)

4. Koutroumbas, K., Theodoridis, S.: Pattern Recognition. Academic Press, London (2006)

5. Randall Wilson, D., Martinez, T.R.: Reduction Techniques for Instance-Based Learning Algorithms, http://citeseer.ist.psu.edu/wilson00reduction.html

6. Hart, P.E.: The Condensed Nearest Neighbor Rule. IEEE Transactions on Information Theory $14,515-516$

7. Ritter, G., Woodruff, H., Lowry, S., Isenhour, T.: An algorithm for a selective nearest neighbor decision rule. IEEE Transactions on Information Theory 21(6), 665-669

8. Gates, G.W.: The Reduced Nearest Neighbor Rule. IEEE Transactions on Information Theory 18(3), 431-433

9. Wilson, D.L.: Asymptotic properties of nearest neighbor rules using edited data. IEEE Transactions on System, Man and Cybernetics 2, 408-421 (1972)

10. Jain, A.K., Nadakumar, K., Ross, A.: Score Normalization in Multimodal Biometrics Systems. Pattern Recognition Letters (2005)

11. Jain, A.K., Dubes, R.C.: Algorithms for Clustering Data. Prentice-Hall, Englewood Cliffs (1988)

12. Jain, A.K., Hong, L., Bolle, R.: On-line Fingerprint Verification. IEEE Transactions on Pattern Analysis and Machine Intelligence 19(4), 302-314 (1997)

13. http://www . equinoxsensors . com/products/HID.html

14. Roli, F., Marcialis, G.L.: Semi-supervised PCA-based face recognition using selftraining. In: Yeung, D.-Y., Kwok, J.T., Fred, A., Roli, F., de Ridder, D. (eds.) SSPR 2006 and SPR 2006. LNCS, vol. 4109, pp. 560-568. Springer, Heidelberg (2006) 